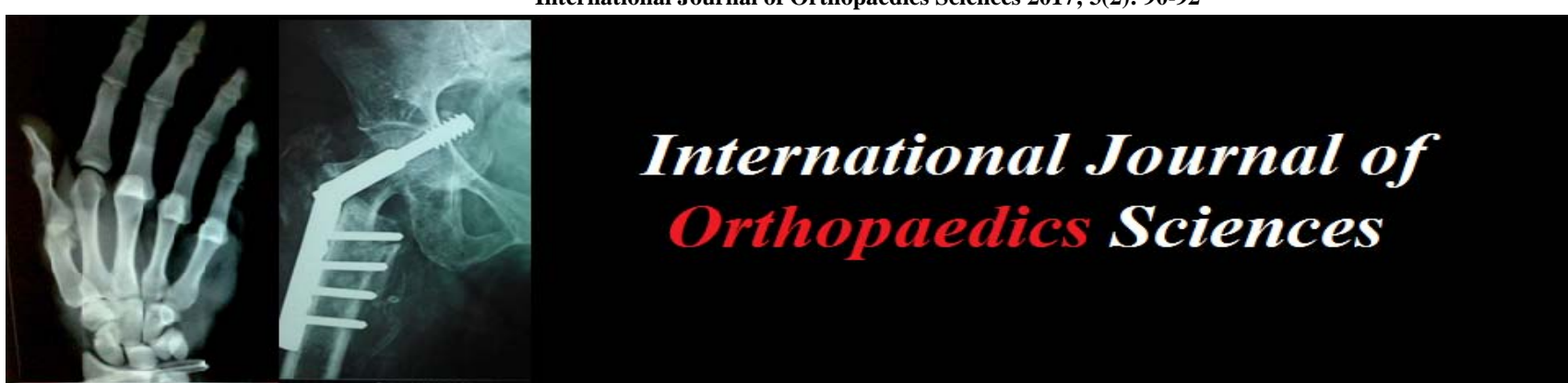

ISSN: $2395-1958$

IJOS 2017; 3(2): 90-92

(C) 2017 IJOS

www.orthopaper.com

Received: 17-02-2017

Accepted: 18-03-2017

Dr. Venkata Suresh Babu

Tummala

Professor, Department of

Orthopaedics, Dr. Pinnamaneni

Siddhartha Institute of Medical

Sciences and Research

Foundation, Chinnaoutpalli,

Krishna DT, Andhra Pradesh,

India

Dr. Suresh Babu Surapaneni

Professor, Department of

Orthopaedics, Dr. Pinnamaneni

Siddhartha Institute of Medical

Sciences and Research

Foundation, Chinnaoutpalli,

Krishna DT, Andhra Pradesh,

India

Dr. Siddarth Pigilam

Former Resident, Department of Orthopaedics, Dr. Pinnamaneni

Siddhartha Institute of Medical

Sciences and Research

Foundation, Chinnaoutpalli, Krishna Dt, Andhra Pradesh, India

Correspondence

Dr. Suresh Babu Surapaneni

Professor, Department of

Orthopaedics, Dr. Pinnamaneni

Siddhartha Institute of Medical

Sciences and Research

Foundation, Chinnaoutpalli, Krishna Dt, Andhra Pradesh, India

\section{Bacteriological study of orthopaedic infections}

\author{
Dr. Venkata Suresh Babu Tummala, Dr. Suresh Babu Surapaneni and \\ Dr. Siddarth Pigilam
}

DOI: $\underline{\text { http://dx.doi.org/10.22271/ortho.2017.v3.i2b.17 }}$

\section{Abstract}

Open fractures are often associated with severe soft tissues damage, devascularization, and devitalization of bone fragments, thus increasing the susceptibility of bone to infection. Infections at the site of orthopaedic implants are also devastating complication. Hence an endeavor is made to study the different bacteria causing orthopaedic infections and susceptibilities of isolated bacteria to various antimicrobial agents. 75 osteomyelitis patients' specimens were collected, bacterial species were isolated and identified by morphology, cultural characteristics and biochemical reactions according to standard techniques and different antibiotics were tested for various microorganisms. Osteomyelitis mainly affects tibia $30.6 \%$ of the cases, more common in males $76 \%$, and was common in 20-39 yrs age group, predominantly of monomicrobial $78 \%$ colonies. Majority of the organisms isolated were Gram positive. Staphylococcus aureus is the commonest pathogen causing Osteomyelitis, among gram negative bacilli Pseudomonas aeruginosa is the commonest isolates. All MRSA strains were $100 \%$ sensitive to vancomycin and linezolid and Gram negative bacteria were sensitive to Cefepime $96 \%$ and Amikacin $70 \%$. From the present study we conclude that Bacteriological study of orthopaedic infections is very much required to treat the osteomyelitis patients with less number of complications and Sequeale.

Keywords: Bacteriological study, osteomyelitis

\section{Introduction}

Bones and joints are normally sterile areas. Bacteria may reach the sites by haematogenous spread or exogenous and endogenous contiguous focus of infection. Osteomyelitis is defined as an infection of bone marrow. Charaka and sushruta ${ }^{[1]}$ quoted about osteomyelitis in their Sanskrit treatises. Hippocrates [500-400 BC] described the bone diseases with injury as the predisposing factor. Hippocrates ${ }^{[2]}$ also described the healing and non-healing factors of bone diseases. The term osteomyelitis was coined by Nelton in 1834.Osteomyelitis results in inflammatory destruction of bone, bone necrosis and new bone formation. Three major categories of osteomyelitis exist, based on pathogenic mechanism of infection. Walvogel 1970 classified Osteomyelitis as, following haematogenous spread, Osteomyelitis secondary to contiguous focus of infection, Osteomyelitis associated with vascular insufficiency. Depending upon the type of infecting organisms, one of the two patterns of host response can occur. Fulminating infections resulting from pyogenic organisms were classically staphylococcus aureus or the gram positive organisms. The second pattern of host response is an insidious granulomatous reaction resulting from less aggressive non pyogenic organisms. In heavily contaminated wounds, gram negative organisms such as Pseudomonas, Salmonella, Klebsiella, Proteus, Escherichia coli may be involved. The foci of infection sometimes develop sinuses which will discharge pus continuously and not abate inspite of administering the best antibiotics. Osteomyelitis can also be of acute or chronic type. Acute type may lead to chronic infection. The state of acute or chronic osteomyelitis depends upon the type of bacteria and host immune response. One of the sequelae of osteomyelitis can be formation of sinuses, sequestrum or involucrum, with resultant morbidity and mortality. Open fractures involve exposure of fracture bone to the extracorporeal environment, thus increasing the risk of bone contamination from foreign debris and bacteria. In addition, open fractures are often associated with severe soft tissues damage, devascularization, and devitalization of bone fragments, thus increasing the susceptibility of bone to infection. Infections at the site of orthopaedic implants are devastating complications, frequently requiring prolonged treatment with multiple 
additional surgical procedures. With increasing use of prosthetic joints, Infections associated with these devices may be the most common and challenging type of septic arthritis encountered by most clinicians. Hence an endeavor is made to study the different bacteria causing orthopaedic infections like Staphylococcus, Klebsiella, Escherichia, Pseudomonas and Proteus etc, their incidence and antibiotic sensitivity pattern. The aim of the present study is to determine the bacteriology of orthopaedic infections and susceptibilities of isolated bacteria to various antimicrobial agents.

\section{Materials and Methods}

The materials for present study collected from 75 osteomyelitis patients admitted in orthopaedic wards of Dr. Pinnamaneni Siddhartha institute of medical sciences \& research foundation, Chinaoutpalli, Gannavaram, from September 2009 to August 2011. The diagnosis of osteomyelitis was based on clinical and radiological findings. Types of specimens collected were Swabs, Aspirated pus, Debridement material, were taken to Microbiology department and Pathology departments for further processing. The swab was inoculated on to nutrient agar, blood agar, Macconkey agar and incubated at 37' $\mathrm{C}$ for 24 hours. Another swab was used for smear preparation; gram staining and histopathological examination. The bacterial species isolated were identified by morphology, cultural characteristics and biochemical reactions according to standard techniques. Antibiotic susceptibility test was done by Kirby Bauer's disc diffusion method. The different antibiotics tested for various microorganisms.

\section{Results}

The present study was undertaken, to identify and isolate the bacterial pathogens from 75 infected patients admitted to orthopaedic units. The specimens collected were swabs $25(33.3 \%)$, debridement material $11(14.6 \%)$ and aspirated pus $39(52 \%)$ for culture, antibiotic sensitivity, gram staining and histopathological examination. Swabs were collected from open fractures, discharging sinus in osteomyelitis, and postoperative wound infections. Debridement was done in chronic osteomyelitis Pus aspirated from acute osteomyelitis and septic arthritis. The specimens collected from different sites were tibia $23(30.6 \%)$ being common, femur $8(10.6 \%)$, humerus $5(6.6 \%)$, fibula $1(1.3 \%)$, hip joint $16(21.3 \%)$, knee joint $8(10.6 \%)$ and other small bones and joints $14(18.6 \%)$. Males 57 (76\%) were affected more than female's i.e.; because the etiology was mainly due to trauma from road traffic accidents. Age wise analysis showed, highest age group affected was 29(20-39 yrs) followed by 22(40-59 yrs), $13(0-19 y r s)$, and $11(60 \&$ above). As the specimens were aerobically cultivated, it was found that there was $50(66.6 \%)$ culture positivity and $25(33.3 \%)$ culture negative cases. Among the isolates, 38(76\%) were monomicrobial and $12(24 \%)$ were polymicrobial (Table 1). The gram positive pathogens isolated were $37(74 \%)$, gram negative pathogen isolated were $20(40 \%)$.

\section{Discussion}

In this bacteriological study of osteomyelitis patients, the specimens collected were swabs, debridement material and aspirated pus from osteomyelitis patient that were admitted. Agarwal et al [3] showed that there was no discrepancy between bacterial flora from sinus tract and sequestrum. Perry et al said that negative culture following inoculation of specimen does not rule out pockets of infection and live bacteria, so numerous cultures from different sites are essential. In the present study specimens collected were 33\% swabs, mainly from open wound fractures, $14.6 \%$ debridement material who were subjected to surgical debridement with chronic osteomyelitis and 52\% pus aspirated from acute osteomyelitis patients. The sample collected were from different bone, tibia 23 (30.6\%) femur 8 $(10.6 \%)$, humerus $5(6.6 \%)$, fibula $1(1.3 \%)$, hip joint $16(21.3 \%)$, knee joint $8(10.6)$ and other small bones $14(18.6 \%)$. Gavilan et al ${ }^{[4]}$ studies shows metaphysis of long bones are involved most commonly tibia 54\%. Ahmed Bo Esia et al in his study concluded that tibia $50 \%$ was the most often involved site of osteomyelitis followed by femur $30 \%$. The percentage of tibial bone involvement correlates well with other studies. A prospective study done by Agarwal et al ${ }^{[3]}$ shows male predominance. Thanni L.O et al ${ }^{[5]}$ shows males were predominant than females. Bonhoeffer $\mathrm{J}$ et al study shows males $61 \%$ and females $39 \%$. In the present study $76 \%$ males predominate females $24 \%$. Distribution of osteomyelitis in different age group showed acute haematogenous was common in school going children. Kao HC. et $a l^{[6]}$ study analyzed that haematogenous osteomyelitis was common in age ranged from 13 to 17 years. Carek P.J et al [7] in his studies shows that acute osteomyelitis was predominant in children and chronic osteomyelitis is usually sub acute that develop secondary to open injury to bone and surrounding soft tissue. Gavilan et al ${ }^{[4]}$ in his study revealed that acute haematogenous infection is more prevalent in children age $0-16$ yrs. In present study acute osteomyelitis was common in 0-19 yrs age group sub acute or chronic osteomyelitis secondary to contagious focus was more predominant in 20-39 yrs age group. Out of 75 cases of osteomyelitis the culture positivity observed in our study was $66.6 \%$, culture positivity revealed by other workers include Gulati, et al $75.5 \%$, Malik F. et al $72.51 \%$, Thanni et al [5] $69.2 \%$. The percentage of culture positivity in our study was on par with culture positivity of various studies conducted by other orthopaedic surgeons and microbiologists. Monomicrobial was seen to be more common then polymicrobial. Zalavaras et al studies show that $70 \%$ were monomicrobial and 30\% polymicrobial, Gulati et al studies showed that monomicrobial $64.5 \%$ and polymicrobial $35.5 \%$, Dendrinos et al reveals that $57.1 \%$ monomicrobial and $42.9 \%$ polymicrobial ${ }^{[8]}$. In present study monomicrobial was $76 \%$ and polymicrobial $24 \%$ correlating well with other studies. Staphylococcus aureus was the commonest organism associated with acute or chronic osteomyelitis. Kao $\mathrm{H}$ et al ${ }^{[6]}$ confirmed this with their study in acute haematogenous osteomyelitis staphylococcus aureus $63 \%$ was the causative agent. Preist et al ${ }^{[9]}$ in his studies found that S. aureus $74 \%$ was isolated mostly. Gavilan M. G. et al ${ }^{[4]}$ his studies show that S.aureus was the main causative organism in all age groups. Kessler et al. in his study isolated S.aureus in 70\% of the osteomyelitis cases. Alonge T. O et al in his study shows that $S$. aureus $78.3 \%$ was the causative organism. According to Thinnai L.O. et al ${ }^{[5]}$ isolated S. aureus $64.7 \%$, gram negative bacterial $34.7 \%$ and tuberculous arthritis $8 \%$. In bacterial study of osteomyelitis by Gulati et al shows that $43.2 \%$ S. aureus, Ps. aeruginosa $18.7 \%$, Proteus $11.7 \%$ Klebsiella and E.coli 9.3\%, staphylococcus epidermidis 1.5\%, streptococcus pyogenes and Enterocci $1.2 \%$. In present study S. aureus $61.39 \%$ isolated followed by Ps. aeruginosa $15.78 \%$ Escherichia coli $1.75 \%$, Klebsiella $7.01 \%$, Enterobacter $3.50 \%$, and Proteus $1.75 \%$ finding that correlate well with other reports showed. In treatment of osteomyelitis 
identification and isolation of pathogens plays a pivotal role in order to prescribe appropriate antibiotics. In present study the antibiotic discs used in susceptibility testing were routinely used in microbiology department of Dr. Pinnamaneni Siddhartha institute of medical sciences \&research foundation. Newer pencillins like carbenicillin, pipericillin imipenem, carbapenem are not used routinely because of the fact that most of the patients cannot afford them. The sensitivity of S.aureus for cephalosporin and quinolones was tested. The study by Jones M. E et al on antibiotic susceptibility demonstrated on narrow spectrum antimicrobials such as vancomycin, linezolid were active against $100 \%$ of isolates. Cephalosporins represented by ceftriaxone, cefotaxime and cefepime exhibited broad spectrum of activity, on Enterobacteriaceae, streptococci and methicillin susceptible $S$. aureus (MSSA) but were not active against methicillin resistance S.aureus (MRSA). The $3^{\text {rd }}$ generation cephalosporins provide broad spectrum coverage was useful for bone infections. According to the study done by Preist D. H. et al ${ }^{[9]}$ isolated $72.5 \%$ Staphylococcus aureus of these $32.5 \%$ were MRSA strains. Gualti et al studies shows that out of $111 \mathrm{~S}$. aureus isolates methicillin resistance was seen in $27.9 \%$. MRSA were $100 \%$ and $96.8 \%$ sensitive to vancomycin and linezolid respectively. Among gram negative organism sulbactam with cefaperazone $89.5 \%$ was most effective followed by amikacin $60.3 \%$. Veenet et al studies show that $46.67 \%$ were identified as MRSA from osteomyelitis and found to be $100 \%$ sensitive to vancomycin. In present study the $S$. aureus were most sensitive to methicillin $72.1 \%$, followed by cefotaxime $70 \%$, and ofloxacin $67.4 \%$. The methicillin resistant Staphylococcus aureus was $27.1 \%$. The MRSA isolates were $100 \%$ sensitive to vancomycin and $87.5 \%$ to clindamycin. S. aureus showed $20 \%$, sensitivity to penicillin. The gram negative bacteria isolate sensitive to $96 \%$ cefepime, $71.8 \%$ cefotaxime and $70.7 \%$ amikacin. The gram negative isolates demonstrated $90 \%$ resistant to ampicillin. The 3rd and 4th generation cephalosporins and amikacin has showed good activity against gram negative bacteria.

\section{Conclusion}

75 Osteomyelitis patients were studied to determine the bacteriology of orthopaedic infections and susceptibilities of isolated bacteria to various antimicrobial agents. Osteomyelitis mainly affects tibia $30.6 \%$ of the cases followed by hip joint, knee joint, femur, humerus and other small bones. More common in males $76 \%$ the reason is trauma road traffic accidents. Osteomyelitis was common in 20-39 yrs age group. Monomicrobial $76 \%$ more predominant than polymicrobial. The organisms isolated were Gram positive in $74 \%$ and Gram negative in $40 \%$. Staphylococcus aureus is the commonest pathogen causing Osteomyelitis. Among gram negative bacilli Pseudomonas aeruginosa is the commonest isolates. Pseudomonas aeruginosa showed sensitivity to $93 \%$ cefepime and $71.2 \%$ to Cefperazone. Staphylococcus aureus was most sensitive to methicillin $72 \%$ and cefotaxime $70 \%$. In all MRSA strains were $100 \%$ sensitive to vancomycin and linezolid. Gram negative bacteria were sensitive to Cefepime $96 \%$ and Amikacin 70\%. From the present study we conclude that Bacteriological study of orthopaedic infections is very much required to treat the osteomyelitis patients with less number of complications and Sequeale.
Table 1: Bacterial Isolates

\begin{tabular}{|c|c|c|c|}
\hline Cultured organisms & \multicolumn{2}{|c|}{$\begin{array}{c}\mathbf{5 0} \% \text { Patients whose } \\
\text { culture positive }\end{array}$} & $\begin{array}{c}\text { Percen } \\
\text { tage }\end{array}$ \\
\hline Staphylococcuc aureus MSSA & 17 & $+\mathrm{ve}$ & 29.82 \\
\hline Staphylococcus aureus MRSA & 18 & $+\mathrm{ve}$ & $31.57 \%$ \\
\hline Escherichia coli & 1 & $-\mathrm{ve}$ & $1.75 \%$ \\
\hline Klebsiella pneumonia & 4 & $-\mathrm{ve}$ & $7.01 \%$ \\
\hline Proteus mirabilis & 1 & $-\mathrm{ve}$ & $1.75 \%$ \\
\hline Enterobacter & 2 & $-\mathrm{ve}$ & $3.50 \%$ \\
\hline Streptococci & 1 & $+\mathrm{ve}$ & $1.75 \%$ \\
\hline Acetobacter & 3 & $-\mathrm{ve}$ & $5.26 \%$ \\
\hline Pseudomonas aeruginosa & 9 & $-\mathrm{ve}$ & $15.78 \%$ \\
\hline Enterococci & 1 & $+\mathrm{ve}$ & $1.75 \%$ \\
\hline
\end{tabular}

\section{References}

1. Charaka and Sushrutha, Sushruta Samhita avurvedic medicine, chapters. 11,145-146.

2. Hippocrate, Oeuvres. Paris: Les Belles Lettres. 19671969.

3. Agarwal G, Kapil A, Kabra SK, Chandra R, Das B, Dwivedi SN. Characterization of Pseudomonas aeruginosa isolated from chronically infected children with cystic fibrosis in India. BMC Microbiology, 2005; 5:43.

4. Gavilán MG, López JB, Artola BS. Peculiarities of osteoarticular infections in children, Baillieres Best Pract Res Clin Rheumatol. 1999; 13(1):77-94.

5. Thanni LOA, Kehinde OA. Trauma at a Nigerian teaching hospital: pattern and documentation of presentation. African Health Sci. 2006; 6(2):104-107.

6. Kao HC, Huang YC, Chiu CH, Chang LY, Lee ZL, Chung PW et al. Acute hematogenous osteomyelitis and septic arthritis in children. J Microbiol Immunol Infect. 2003; 36:260-265.

7. Carek PJ, Dickerson LM, Sack JL. Diagnosis and management of osteomyelitis. Am Fam Physician 2002; 65(9): 1751.

8. Dendrinos GK, Kontos S, Lyritsis E. Use of the Ilizarov technique for treatment of non-union of the tibia associated with infection. J Bone Joint Surg Am. 1995; 77(6):835-846.

9. Priest DH, Peacock JE jr, studies on haematogenous vertebral osteomyelitis due to Staphylococcus aureus in adults South Med. J. 2006; (9):853. 